\title{
Recent Advances in Diagnosis, Prevention and Treatment of Neonatal Sepsis
}

\author{
Tanushri Mukherjee ${ }^{1}$, Sanjay Wazir ${ }^{2}$
}

\begin{abstract}
Sepsis is one of the most common causes of morbidity and mortality in the neonatal period. Although this is a global problem, the magnitude of the problem is huge in developing countries because of a lack of clear guidelines for the management of the condition, lack of standard laboratory services and impulsivity of the clinician in changing the antibiotics for any slight deterioration which can be explained by other noninfectious conditions. The source of infection in the baby can be from the mother called the early onset sepsis or from the community or hospital called the late-onset sepsis. Proper identification and knowledge of the causative microorganism can help decide the right antibiotic and causative microorganisms in one area or hospital would be different from the other area and hence it is prudent to make efforts for the identification of the organism causing the disease. Treatment requires the judicious use of antibiotics with the proper choice of antibiotics as well as proper dose and duration. Overuse of antibiotics should be discouraged because of risk of complications and resistance development. Keywords: Adjuvant treatment, Antibiotics, Developing countries, Early onset sepsis, Empiric treatment, Late-onset sepsis, Neonate. Pediatric Infectious Disease (2019): 10.5005/jp-journals-10081-1213
\end{abstract}

Infection is one of the major reasons of mortality and morbidity in neonates and is directly related to increased duration of hospital stay amongst them. The problem is worse in developing countries where the maximum number of births happen. Neonates are more prone to infections because of the immature immune system and the problem is more at lesser gestations because of the lack of transfer of maternal antibodies in the last trimester. The problem is compounded by the presence of many coexisting neonatal illnesses during this time with overlapping symptoms. Despite being a single diagnosis, etiological agents, therapeutic management strategy and the controlling mechanism would vary from one region to another in the world.

\section{EPIDEMIOLOGY}

It is estimated that approximately 3 million cases of neonatal sepsis occur every year globally. ${ }^{1}$ According to the WHO (World Health Organization) neonatal sepsis was responsible for an estimated 430,000 neonatal deaths in 2013 , accounting for approximately $15 \%$ of all neonatal deaths in the world. ${ }^{2}$

Incidence of neonatal sepsis varies significantly between developing and developed countries. The overall incidence of sepsis in western countries ranges between $1 / 1,000$ and $5 / 1,000$ live births and is inversely related to the gestational age. Data was collected from academic institutions with tertiary NICUs from India as part of National Neonatal Perinatal Database (NNPD), ${ }^{3}$ and the reported incidence of sepsis was 38/1,000 live births. In a recent study, amongst the neonates admitted to tertiary-level NICUs in different hospitals in northern India, blood culture positivity was seen in $13.1 \%$ cases and total sepsis which includes all cases (probable and culture proven cases) was reported in $54.7 \%$ cases. ${ }^{4}$ In community settings, the date is less alarming but still a significant burden exists with the incidence of culture-positive being 12.3 per 1,000 live births. This data is largely influenced by Aetiology of Neonatal infections in South Asia (ANISA) study, which used advanced microbiology techniques and unique statistical models in reaching the results. ${ }^{5}$
${ }^{1}$ Department of Neonatology and Pediatrics, Cloudnine Hospital, Mumbai, Maharashtra, India

${ }^{2}$ Department of Neonatology and Pediatrics, Cloudnine Hospital, Gurugram, Haryana, India

Corresponding Author: Sanjay Wazir, Department of Neonatology and Pediatrics, Cloudnine Hospital, Gurugram, Haryana, India, Phone: +919910076765, e-mail: swazir21@gmail.com

How to cite this article: Mukherjee T, Wazir S. Recent Advances in Diagnosis, Prevention and Treatment of Neonatal Sepsis. Pediatr Inf Dis 2019;1(3):108-113.

Source of support: Nil

Conflict of interest: None

\section{Microorganisms Involved}

In western countries, group B Streptococcus (GBS) is the most common responsible organism for early-onset sepsis (EOS). In late-onset sepsis (LOS), Staphylococcus aureus and CONS (coagulasenegative staphylococci) are the most common organisms.

However, according to the NNPD data, ${ }^{3}$ in Indian scenarios, Klebsiella (23\%), E. coli (14\%), and Acinetobacter (8\%) accounted for the most common organisms responsible for neonatal sepsis. The organism pattern was similar between EOS and LOS. S. aureus (20\%) and CONS (9\%) were the most common gram positive organisms. Klebsiella (25\%), E. coli (15\%), and S. aureus (12\%) were the common organisms even amongst the samples from the community settings. The similarity between the EOS and LOS organisms in Indian scenarios in the hospital settings is suggestive of the fact that most infections are due to improper hygiene practices in the delivery room.

The common pathogens from NNPD data show a high degree of resistance to first-line antibiotics being used in the western world, i.e., ampicillin and gentamicin. ${ }^{3}$ Third-generation cephalosporins

(c) The Author(s). 2019 Open Access This article is distributed under the terms of the Creative Commons Attribution 4.0 International License (https://creativecommons. org/licenses/by-nc/4.0/), which permits unrestricted use, distribution, and non-commercial reproduction in any medium, provided you give appropriate credit to the original author(s) and the source, provide a link to the Creative Commons license, and indicate if changes were made. The Creative Commons Public Domain Dedication waiver (http://creativecommons.org/publicdomain/zero/1.0/) applies to the data made available in this article, unless otherwise stated. 
(cefotaxime) resistance is also quite high. Fifty percent of S. aureus isolates were also resistant to methicillin. However, the data from the community suggests resistance to cefotaxime and gentamicin was low but was high for ampicillin. The high antimicrobial resistance suggests that there is an unregulated use of antibiotics in neonates, especially those with culture -ve sepsis.

\section{Classification and Terminology}

The commonly used terminologies in neonatal sepsis include the following:

\section{Clinical or Probable Sepsis}

In a neonate having symptoms suggestive of sepsis and one of the following:

- Presence of factors like maternal fever and foul-smelling liquor.

- X-ray suggestive of pneumonia.

- Positive sepsis screen.

\section{Culture-positive Sepsis}

Isolation of a microorganism from normally sterile site like blood, cerebrospinal fluid (CSF), and urine in a neonate with clinical features suggestive of sepsis.

\section{Early-onset Sepsis}

Presenting within the first 3 days of birth with fulminant respiratory or multisystem illness due to bacteria acquired during birth or having spread from the vaginal canal.

\section{Late-onset Sepsis}

LOS usually presents after 72 hours of life. The infection can come from either the community or the hospital, and presents with septicemia, meningitis, or pneumonia.

\section{Risk Factor-based Approach}

One of the major problems at birth is to decide if the neonate would require empirical antibiotics. For this reason, some of the clinicians have resorted to using a combination of risk factors for estimating this risk of neonatal sepsis in babies at birth. For EOS, one study, done at Chandigarh, ${ }^{6}$ looked at the various risk factors and their importance in deciding the treatment plan (Table 1).

The purpose is to help the clinician guide the initiation of presumptive antibiotics. The study validated that one is justified in starting antibiotics if the scores are more than 6 even if the neonate is asymptomatic. In cases of clinical chorioamnionitis (defined as maternal fever more than 100.4 F and one of the following: maternal tachycardia, maternal total leucocyte count (TLC), fetal tachycardia, uterine tenderness, and foul-smelling liquor), antibiotics should be started irrespective of the score. For a score of less than 6 , it is prudent to do a limited evaluation with complete blood count (CBC)

Table 1: Risk factors for early-onset sepsis and their relative importance

\begin{tabular}{ll}
\hline Risk factor & Score \\
\hline Birth weight $<1,500 \mathrm{~g}$ & 3 \\
Gestation $<30$ weeks & 2 \\
Per vaginal examinations $\geq 3$ & 6 \\
Intrapartum antibiotics not received & 2 \\
Male gender & 3 \\
Clinical chorioamnionitis & 6 \\
\hline
\end{tabular}

and blood culture at 6-12 hours and then start antibiotics only if the neonate becomes symptomatic or screening results are abnormal.

\section{Clinical Presentation}

The manifestations of neonatal sepsis are very nonspecific and can mimic any other neonatal morbidities usually present in the neonatal period. Therefore, the diagnosis of sepsis must be considered in the differential diagnosis of any clinical worsening unless the picture can be fully explained by other causes.

The clinical manifestations of neonatal sepsis often have multiorgan involvement but one or the other organ system symptoms may predominate (Table 2 ).

The signs are similar in both preterm and term neonates, but the index of suspicion needs to be very high for preterms since many symptoms like apnea, decreased activity, tachycardia may be seen in other preterm morbidities as well. Sclerema, shock, disseminated intravascular coagulation and pulmonary hemorrhage are signs of fulminant sepsis.

\section{Diagnosis}

Since the features of neonatal sepsis are very nonspecific, definitive diagnosis requires the isolation of a microorganism from either from blood, CSF, or urine (which are normally sterile). Other markers of inflammation like sepsis screen are often used for aiding the diagnosis of neonatal sepsis. Various tests which aid the diagnosis are listed below.

\section{Complete Blood Count}

This is the most common investigation done in neonatal sepsis across the NICUs. However, because of the low sensitivity, it cannot be used to establish a diagnosis of sepsis. Its use under two scenarios in EOS and LOS are described below.

In EOS, it is a good idea to do the $C B C$ at 6-12 hours after birth as those values are more predictive of sepsis than those obtained immediately after birth because the white blood cell (WBC) and absolute neutrophil count (ANC) normally increase during the first 6 hours of life. ${ }^{7}$ Based on two large multicentric studies, a low WBC count $(<5,000 / \mu \mathrm{L})$, ANC $(<1,000$ neutrophils $/ \mu \mathrm{L})$, and elevated I/T ratio are generally associated with proven sepsis but lack sufficient sensitivity and specificity to be used for diagnosing sepsis. Although both elevated and low neutrophil counts can be associated with neonatal sepsis, neutropenia has greater specificity as few conditions other than sepsis and preeclampsia depress the neutrophil count of neonates. ${ }^{7,8}$

Table 2: Organ-specific manifestations of neonatal sepsis

\begin{tabular}{ll}
\hline System & Clinical feature \\
\hline General & $\begin{array}{l}\text { Lethargy, hypothermia, hyperthermia, poor } \\
\text { feeding and increasing jaundice }\end{array}$ \\
Respiratory & $\begin{array}{l}\text { Respiratory distress after a period of normalcy } \\
\text { (usual but not always) } \\
\text { Hypotension and tachycardia }\end{array}$ \\
Cardiac & $\begin{array}{l}\text { Feeding intolerance, abdominal distension, } \\
\text { vomiting and diarrhea }\end{array}$ \\
CNS & $\begin{array}{l}\text { Irritability, seizures and full anterior fontanelle } \\
\text { Skin }\end{array}$ \\
Metabolic & $\begin{array}{l}\text { Metabolic acidosis, hyperglycemia and } \\
\text { hypoglycemia }\end{array}$ \\
Hematological & $\begin{array}{l}\text { Bleeding from the puncture sites or mucosal } \\
\text { bleeding }\end{array}$ \\
\hline
\end{tabular}


The lower limit of WBC for different gestations at 6-8 hours of birth (when ideally the CBC should be done for evaluation) is as following: at $>36$ weeks of gestation, $7,500 / \mu \mathrm{L}$ and for neonates born at 28 through 36 weeks of gestation, 1,500/ $\mathrm{L}^{.}{ }^{9}$

\section{LOS}

In a study of 37,826 neonates with suspected LOS, abnormal WBC $(<1,000$ or $>50,000 / \mu \mathrm{L})$, high ANC $(>17,670 / \mu \mathrm{L})$, elevated $\mathrm{I} / \mathrm{T}$ ratio $(\geq 0.2)$, and low platelet count $(<50,000 / \mu \mathrm{L})$ were associated with culture positivity. ${ }^{8}$ However, sensitivity was inadequate to reliably predict LOS. So this is not a great test to rely on for the diagnosis of LOS.

\section{WBC Surface Markers}

Infection leads to upregulation of cell surface markers and the most commonly studied is CD64 ( $\mathrm{FcyRI})$, a receptor that binds the $\mathrm{Fc}$ portion of gamma immunoglobulin. CD64 expression rises rapidly at the onset of an infection and stays elevated for a minimum of 24 hours and can be measured rapidly in a small amount of blood. However, dependence on flow cytometry precludes the use in routine clinical scenarios and its value may be better in term neonates with EOS rather than other scenarios. This is not routinely available for clinical use in India at present.

\section{CRP}

C-reactive protein (CRP) is an acute-phase reactant secreted by liver in response to a variety of inflammatory conditions including both viral and bacterial infections. A variety of noninfectious neonatal inflammatory conditions can also cause elevated CRP, including perinatal asphyxia, meconium aspiration, intraventricular hemorrhage, immunization and viral infections. The cutoffs to define positivity vary according to the method used and should always be checked with the concerned laboratory. Values more than $10 \mathrm{mg} / \mathrm{L}$ can often be found in normal neonates born by vaginal route especially those needing instrumentation. The increase in values in preterm neonates may not be as high as term neonates with a similar disease. It tends to rise 4-6 hours after the insult and reaches a peak around 36-48 hours with a half-life of 19 hours with $50 \%$ daily reduction in value if the inflammatory stimulus is gone, and hence, if done very early in the sepsis evaluation, the result may be falsely negative.

A single value of CRP does not help make a diagnosis of neonatal sepsis because it lacks sufficient sensitivity and specificity. ${ }^{10}$ Almost $30 \%$ of the neonates in a study with sepsis had a normal CRP at the time of initiation of therapy. Hence, CRP alone should not be used as a marker suggestive of sepsis. However, the sequential assessment of CRP values may help negate a diagnosis of sepsis. If the two sequential CRP levels remain persistently normal $[<1 \mathrm{mg} /$ $\mathrm{dL}(10 \mathrm{mg} / \mathrm{L})]$, neonatal bacterial sepsis is unlikely with almost $99 \%$ negative predictive value. ${ }^{11}$

CRP levels can be helpful in guiding antibiotic duration in suspected neonatal bacterial infection. But continuation of empiric antibiotics for more than 48 hours in well-appearing neonates with negative culture results and an elevated CRP level alone is not justified. ${ }^{12}$ If the CRP values remain persistently elevated, one should look for alternate explanations like complications rather than just continue antibiotics.

\section{Procalcitonin}

Procalcitonin (PCT) is the peptide precursor of calcitonin released by parenchymal cells in response to bacterial toxins. In noninfected neonates, $\mathrm{PCT}$ values increase after delivery, increase to maximum by 24 hours of life and then fall to $<0.5 \mu \mathrm{g} / \mathrm{L}$ by 72 hours of postnatal age. Procalcitonin cutoff level $0.5 \mu \mathrm{g} / \mathrm{L}$ proves useful for the diagnosis of late-onset infection in neonates. If there is no persisting stimulus, PCT is eliminated from the body within 24-36 hours, which makes it useful for monitoring of therapy. NeoPInS is the largest multicentric randomized controlled trial (RCT), which looked at the value of using PCT for antibiotic stewardship in newborns. This trial was conducted across 18 centers in the western world to investigate if PCT could assist in reducing the antibiotic duration in neonates with EOS. ${ }^{13}$ One thousand and seven hundred and ten newborns $>34$ weeks of gestational age were randomized to conventional care or antibiotic duration based on the PCT values. If two PCT values were negative, the antibiotics were stopped, and with this protocol, approximately one-third of neonates did not get prolonged antibiotics and also duration of exposure to antibiotics was also reduced by 22 hours.

Limitations with the use of PCT is that it cannot be used as a sole marker of sepsis because it can get elevated in other noninfectious conditions like intra ventricular hemorrhage (IVH), hypoxic ischemic encephalopathy (HIE), and there is significant variation in the normal values in term and preterm population in the first 72 hours. So, it can be used for deciding when to stop antibiotics. However, for diagnosis of sepsis especially EOS, it is not a good marker.

\section{Cytokines, Chemokines, and Other Biomarkers}

Both proinflammatory cytokines (interleukin- 6 and tumor necrosis factor-alpha) and antiinflammatory cytokines (IL-4 and IL-10) are increased in infected neonates compared with those without infections. ${ }^{14}$ Elevations of serum amyloid $A$ and the cell surface antigen CD64 also have high sensitivity for identifying neonates with sepsis. ${ }^{15}$ However, these biomarkers are not routinely measured because of the cost of testing and lack of sufficient sensitivity to reliably detect neonatal sepsis. ${ }^{14}$

\section{Blood Culture}

The most definitive proof of sepsis is by growing the microorganism in the culture of blood from the neonate. With advancements in technology, the earlier manually read broth-based methods have been replaced with continuously monitored, automated blood culture systems. The ability of a single blood culture to recognize neonatal bacteremia is around $90 \%$.

\section{Time to Positivity}

The growth of bacteria happens in most cases by 48 hours in blood culture bottles. In laboratory experiments, a delay in putting the blood culture bottle into the machine can increase the time for bacteria to grow in the culture bottle. ${ }^{16}$ Hence, it is a good NICU practice to shift the collected blood culture bottle to the laboratory as soon as possible for inoculation in the machine.

Concerns have been raised about the incomplete detection of low-level bacteremia and the effect of intrapartum antibiotic administration on the yield of blood cultures. However, newer optimized enriched culture media with antimicrobial neutralization properties and continuous-read detection systems are used reliably to detect bacteremia at a level of $1-10 \mathrm{CFU} / \mathrm{mL}$, if a minimum blood volume of $1 \mathrm{~mL}$ is inoculated. ${ }^{11}$

\section{Volume of Blood for Inoculation}

The optimal volume of blood needed to detect bacteremia in neonates has not been determined and depends on the organism, 
level of bacteremia, and previous use of antibiotics. Laboratory experiments have shown that common pediatric pathogens can be reliably recovered from $0.5 \mathrm{~mL}$ of blood even when cultured at blood-to-broth ratios of 1:100. ${ }^{17}$ However, in situations of lowlevel bacteremia ( $<10 \mathrm{CFU} / \mathrm{mL}$ of blood), $0.5 \mathrm{~mL}$ of blood is often not sufficient to detect bacteremia. ${ }^{18}$ So, for detecting all levels of bacteremia, at least $1 \mathrm{~mL}$ of blood would need to be inoculated in the blood culture bottle.

\section{Sampling Site}

Blood for culture can be obtained from a venous puncture or a peripheral arterial puncture or from a newly inserted central vascular catheter without flushing it with any solution. Indwelling catheters are usually colonized with microorganisms, and hence, culture results from these sites are difficult to interpret. Similarly, the samples or capillary blood may suggest the skin contaminant rather than the true infection. If catheter-associated sepsis is suspected, a culture should be obtained through the catheter as well as through a peripheral vein.

\section{Number of Culture Bottles to be Inoculated}

One blood culture bottle is generally enough prior to starting empirical antibiotics in neonates. The use of two separate blood culture bottles may help exclude common skin contaminants especially CONS and help differentiate between contamination and true infection. In one study, it was shown that in $5 \%$ of neonates if a subsequent blood culture failed to validate the presence of CONS, it could help reduce antibiotic usage by $8 \% .{ }^{19}$ Anerobic cultures are generally not required. We commonly use only one bottle for blood culture but use the second one if the first culture grew CONS and the neonate is asymptomatic.

\section{Distinguishing Infection from Contamination}

Increased use of intravascular catheters can cloud the interpretation of results especially in conditions where the symptoms are very mild or none at all. Some of the following factors could be considered while differentiating infection from contamination.

- Positive blood culture from a peripheral vein is confirmatory of sepsis when a known pathogenic bacteria is detected in a symptomatic neonate.

- If the culture takes a longer time $>3$ days to grow in a neonate with mild symptoms, then contamination is likely.

- If the neonate improves within 4-6 hours of deterioration, it is unlikely because of infection. If an alternate cause has been found, repeat a culture from the peripheral line.

- If multiple organisms have grown, or different organisms grown in two bottles (if sent) or known skin flora (e.g., diphtheroids), then contamination is more likely.

- If two cultures one from the peripheral line and other from the central line show the same organism in an asymptomatic neonate, it is most likely to represent true infection.

- In case of culture taken only from the indwelling line is positive in a neonate with some symptoms, then one should repeat a culture within 24 hours from a peripheral line and if that is also positive, then treat as true infection.

\section{Lumbar puncture is indicated in the following situations}

- All cases undergoing evaluation for sepsis.

- Positive blood culture cases. However, one must realize that blood culture could be negative in up to $38 \%$ of cases of meningitis. ${ }^{20}$
Table 3: CSF values of cytology and biochemistry in defining sepsis

Amongst neonates Amongst neonates with blood culture with suspected sepsis positive sepsis

\begin{tabular}{lll}
\hline $\begin{array}{l}\text { Preterm babies } \\
\begin{array}{l}\text { Cutoff values to } \\
\text { diagnose meningitis }\end{array}\end{array}$ & $\begin{array}{l}>170 \text { or WBC }>100 \text { or } \\
\text { glucose }<25\end{array}$ & $<25$ or protein $>170$ \\
Term babies & WBC $>21$ or & WBC $>8$ or glucose \\
& glucose $<20$ & $<20$ or protein $>120$
\end{tabular}

- Laboratory data suggestive of sepsis for which alternate diagnosis is not available.

- Worsening clinical status while on antibiotic therapy.

Amongst those with only risk factors and no symptoms, the incidence of meningitis is very low $(<1 \%)$, and hence, they do not require an lumbar puncture (LP). ${ }^{21}$ If the clinical condition can be explained by other noninfectious causes such as respiratory distress (RDS) and transient tachypnea of newborn (TTNB), one could hold on to do the lumbar puncture especially if you are not planning to start antibiotics. ${ }^{22}$ In an unstable neonate, the LP can be deferred, until stabilization is achieved.

The values of CSF cytology, protein content and the sugar which signifies infections have always been a controversial issue. We here present the recommendations of the National Neonatology Forum (NNF) clinical guidelines 2011 to define meningitis in the neonate (Table 3).

\section{Urine Culture}

The signs of urinary tract infection (UTI) in neonates are nonspecific and varied. Urine culture must always be performed on neonates being evaluated for sepsis more than 7 days of life on a sample obtained by a suprapubic puncture or by a fresh bladder catheterization. A cutoff of $\geq 10 \mathrm{WBCs} / \mathrm{mL}$ cells per cubic $\mathrm{mm}$ in uncentrifuged urine has a sensitivity of $82 \%$, specificity $94 \%$ in the diagnosis of neonatal sepsis.

\section{Management}

\section{Antibiotic Stewardship in Sepsis}

Neonates may be exposed to antibiotic drugs before birth in the form of GBS IAP (intrapartum prophylaxis), maternal surgical prophylaxis in cesarean deliveries, intra-amniotic infection or other maternal infections. Retrospective data from the term neonates have linked the antibiotic usage in neonatal period with dangers of later childhood medical issues like wheezing, asthma, food allergies, inflammatory bowel disease, childhood obesity and alteration of developing gut microbiome. ${ }^{23}$ Hence, the use of antibiotics should be rationalized in neonates.

Currently most premature neonates are empirically treated with antibiotics often for prolonged periods, even in the absence of a culture-confirmed infection. Empirical antibiotics given to very preterm neonate in the first days after birth have been associated with an increased likelihood of death, necrotizing enterocolitis (NEC) and bronchopulmonary dysplasia (BPD). ${ }^{24}$

Principles of antibiotic stewardship program:

- Enhancing infection prevention and control.

- Prescribing antibiotics when they are truly needed. 
- Prescribing appropriate antibiotics and in appropriate doses.

- Using the shortest possible duration of antibiotics based on evidence.

- Reviewing the treatment once the culture is available.

- Surveillance of antimicrobial resistance and audit of antibiotic usage.

Indications for empiric antibiotic therapy include the following:

- Sick-appearing neonates.

- Symptomatic neonates: temperature instability, respiratory, cardiocirculatory, and neurologic symptoms.

- CSF pleocytosis (WBC >20-30 cells/ $\mu \mathrm{L}$ ).

- Confirmed or suspected maternal chorioamnionitis.

- Positive blood, urine, or CSF culture.

\section{Initial Empirical Therapy}

The initial choice of parenteral antimicrobials for suspected sepsis is based on the age, likely pathogens, the susceptibility patterns of organisms in a particular nursery and the presence of an apparent source of infection. It is a good idea to construct the antibiotic policy based on the blood culture and drug sensitivity report of last year. The first-line antibiotic chosen should be able to cover approximately $70 \%$ of the prevalent organisms in the last year. Second-line antibiotic (single or combination) should cover approximately $90 \%$ of the prevailing organism and third-line antibiotics should be able to cover for $95-100 \%$ of the organisms.

As discussed earlier, since the organisms responsible for EOS/ LOS in India are similar and hence empirical antibiotics in both situations should be similar.

\section{Special Circumstances}

- Suspected meningitis - in neonates with LOS with meningitis (e.g., CSF pleocytosis), cefotaxime, or ceftazidime is added because of better CNS penetration of these drugs. Meropenem is preferred if there is a concern for infection due to multidrugresistant gram-negative organisms.

- Suspected pneumonia-ampicillin, gentamicin or ampicillin, cefotaxime or vancomycin, and gentamicin to covet for Listeria.

- Skin, soft tissue, bone, and joint infections-vancomycin or nafcillin to cover for Staphylococcus.

- Catheter-related infection-vancomycin and gentamicin.

- Suspected intestinal source-clindamycin or metronidazole to account for anaerobes.

\section{Upgradation of Empirical Antibiotics}

Escalation of therapy must be done in case the expected clinical improvement does not occur in 48-72 hours or any new sign appears. Frequent and very early changes are likely to result in antibiotic resistance development.

In the case of the extremely sick neonate or deteriorating very rapidly, the first line of antibiotics can be bypassed and one can directly start with the 2 nd line of antibiotics.

Once the blood culture report is available, narrower spectrum and lower cost pathogen-specific monotherapy should be used.

\section{Special Considerations}

If the empirical antibiotics are reported sensitive but the neonate has worsened on these antibiotics, it may be a case of in vitro resistance. Antibiotics may be changed to an alternate sensitive antibiotic with the narrowest spectrum and lowest cost.
- If the empirical antibiotics are reported resistant but the neonate has improved clinically, it may or may not be a case of in vivo sensitivity. In such cases, careful assessment must be made before deciding on continuing with the empirical antibiotics.

- One must not continue with antibiotics with in vitro resistance in case of Pseudomonas, Klebsiella, and Methicillin resistant Staphylococcus aureus (MRSA), and in cases of central nervous system (CNS) infections and deep-seated infections.

- If no antibiotic has been reported sensitive but one or more has been reported "moderately sensitive," therapy must be changed to such antibiotics at the highest permissible dose. It is preferable to use a combination of two.

\section{Duration of Antibiotics}

- Blood culture positive: 10-14 days.

- Meningitis, culture positive: GBS or gram-positive organisms, such as L. monocytogenes or Enterococcus, 14 days. E. coli or other gram-negative enteric pathogens, 21 days.

- For neonates with CSF pleocytosis and bacteremia, but a negative CSF culture continue meningeal doses of antimicrobial therapy for 10 days for gram-positive bacteremia and 14 days for gram-negative bacteria.

- Bone and joint infection, ventriculitis: 6 weeks

- UTI: 7-14 days

\section{Lumbar Puncture Delayed}

For neonates in whom the CSF evaluation was delayed because of clinical instability, meningeal doses of antimicrobial therapy should be continued until the LP can be safely performed.

The total duration of therapy depends upon the CSF evaluation and blood culture result:

- Those who have a CSF pleocytosis and positive blood culture treated for 10 days for gram-positive bacteremia (e.g., GBS) and 14 days for gram-negative bacteremia.

- For those who have a CSF pleocytosis and negative blood culture, duration of meningitic doses of antimicrobial therapy based on clinical parameters.

- For those who have a normal CSF profile, negative blood, and CSF cultures, discontinue antimicrobial therapy when cultures are sterile after $48-72$ hours.

\section{Adjunct Therapy to Antibiotics}

A variety of adjunctive immunotherapeutic interventions has been evaluated, but none have been shown to conclusively improve the outcome of neonates with sepsis.

- Intravenous immunoglobulin: the most recent 2013 Cochrane analysis which included 19 randomized controlled trials with approximately 5,000 neonates less than 37 weeks and less than $2,500 \mathrm{~g}$ in birth weight for prevention of neonatal sepsis ${ }^{25}$ concluded that IVIG was not indicated for prophylaxis in this group as there was no effect on long-term and short-term outcomes like mortality. Cochrane analysis involving 3,871 neonates included who received adjunct therapy with IVIG for suspected or proven bacterial/fungal infection, compared with placebo or no intervention, and also included the famous INIS study (the largest study involving 3,493 neonates enrolled in 113 hospitals in nine countries) also concluded that there was no significant difference in mortality. Based on the cumulative data, adjunctive therapy with IVIG is not recommended in the management of neonatal sepsis. ${ }^{26}$ 
- Colony stimulating factors: in a systematic review, the routine use of G-CSF and GM-CSF did not reduce mortality of neonatal sepsis. ${ }^{27}$ In addition, the prophylactic use of growth factors did not reduce the risk of systemic infection in high-risk patients. A subset analysis from three of these studies that included 97 neonates with neutropenia (ANC less than 1,700 per $\mu \mathrm{L}$ ) showed an apparent reduction in mortality ( $\mathrm{RR}, 0.34 ; 95 \% \mathrm{Cl}, 0.12-0.92)$ but the numbers are too small to draw any meaningful conclusion. From the Cochrane review, ${ }^{27}$ there is no evidence that the addition of granulocyte-CSF (G-CSF) or granulocyte monocyte-CSF (GMCSF) to antibiotic therapy in preterm neonates with suspected systemic infection reduces mortality. Hence, the data suggest that G-CSF for neonatal sepsis is safe and may be useful in some special situations but routine use is not warranted.

- Pentoxifylline: a xanthine derivative inhibits the release of tumor necrosis factor (TNF)-alpha, which is generally associated with systemic gram-negative infection. Low-quality data from the meta-analysis of seven trials suggest that pentoxifylline therapy was associated with a decrease in all-cause mortality during hospital stay (RR $0.57,95 \% \mathrm{Cl} 0.35-0.93){ }_{i}^{28}$ however, more data would be required before recommending the same in routine use.

\section{Conclusion}

Neonatal sepsis is one of the most significant causes of mortality early on in life and all efforts should be made to prevent this dreaded disease. A systematic approach to evaluation of the disease including a risk-based approach and proper use of diagnostic tests is required for controlling both over and under diagnosis of this condition. Antibiotics should be used in proper dose and for proper duration to treat this condition. Overuse can lead to complications, and resistance and early discontinuation where it is not required to be continued should be the aim.

\section{References}

1. Fleischmann-StruzekC, Goldfarb DM, Schlattmann P, et al. The global burden of paediatric and neonatal sepsis: a systematic review. Lancet Respir Med 2018;6(3):223-230. DOI: 10.1016/S2213-2600(18)30063-8.

2. Oza S, Lawn JE, Hogan DR, et al. Neonatal cause-of-death estimates for the early and late neonatal periods for 194 countries: 2000-2013. Bull World Health Organ 2015;93(1):19-28. DOI: 10.2471/BLT.14.139790.

3. Report of the National Neonatal Perinatal Database. Report 20022003. NNPD Network. 2005 Jan; http://www.newbornwhocc.org/ pdf/nnpd_repo rt_2002-03.pdf.

4. Jajoo M, Manchanda V, Chaurasia S, et al. Alarming rates of antimicrobial resistance and fungal sepsis in outborn neonates in North India. PLoS One 2018;13(6):e0180705. DOI: 10.1371/journal. pone. 0180705 .

5. Saha SK, Schrag SJ, El Arifeen S, et al. Causes and incidence of community-acquired serious infections among young children in south Asia (ANISA): an observational cohort study. Lancet 2018;392(10142):145-159. DOI: 10.1016/S0140-6736(18)31127-9.

6. Dutta S, Reddy R, Sheikh S, et al. Intrapartum antibiotics and risk factors for early onset sepsis. Arch Dis Child Fetal Neonatal Ed 2010;95(2):F99-F103. DOI: 10.1136/adc.2009.163220.

7. Newman TB, Puopolo KM, Wi S, et al. Interpreting complete blood counts soon after birth in newborns at risk for sepsis. Pediatrics 2010;126(5):903-909. DOI: 10.1542/peds.2010-0935.

8. Hornik CP, Benjamin DK, Becker KC, et al. Use of the complete blood cell count in early-onset neonatal sepsis. Pediatr Infect Dis J 2012;31(8):799-802. DOI: 10.1097/INF.0b013e318256905c.

9. Schmutz N, Henry E, Jopling J, et al. Expected ranges for blood neutrophil concentrations of neonates: the Manroe and Mouzinho charts revisited. J Perinatol 2008;28(4):275-281. DOI: 10.1038/ sj.jp.7211916.
10. Pourcyrous $M$, Bada HS, Korones SB, et al. Significance of serial C-reactive protein responses in neonatal infection and other disorders. Pediatrics 1993;92(3):431-435.

11. Puopolo KM, Benitz WE, Zaoutis TE, et al. Management of neonates born at $\geq 350 / 7$ weeks' gestation with suspected or proven earlyonset bacterial sepsis. Pediatrics 2018;142(6):e20182894. DOI: 10.1542/ peds.2018-2894.

12. Benitz WE, Wynn JL, Polin RA. Reappraisal of guidelines for management of neonates with suspected early-onset sepsis. J Pediatr 2015;166((4):1070-1074. DOI: 10.1016/j.jpeds.2014.12.023.

13. Stocker M, van Herk W, El Helou S, et al. Procalcitonin-guided decision making for duration of antibiotic therapy in neonates with suspected early-onset sepsis: a multicentre, randomised controlled trial (NeoPIns). Lancet 2017;390(10097):871-881. DOI: 10.1016/S01406736(17)31444-7.

14. Zhou M, Cheng S, Yu J, et al. Interleukin-8 for diagnosis of neonatal sepsis: a metaanalysis. PLoS One 2015;10(5):e0127170. DOI: 10.1371/ journal.pone.0127170.

15. Hedegaard SS, Wisborg K, Hvas AM. Diagnostic utility of biomarkers for neonatal sepsis--a systematic review. Infect Dis (Lond) 2015;47(3):117-124. DOI: 10.3109/00365548.2014.971053.

16. Jardine LA, Sturgess BR, Inglis GD, et al. Neonatal blood cultures: effect of delayed entry into the blood culture machine and bacterial concentration on the time to positive growth in a simulated model. J Paediatr Child Health 2009;45(4):210-214. DOI: 10.1111/j.14401754.2008.01455.x.

17. Kennaugh JK, Gregory WW, Powell KR, et al. The effect of dilution during culture on detection of low concentrations of bacteria in blood. Pediatr Infect Dis 1984;3(4):317-318. DOI: 10.1097/00006454198407000-00008.

18. Schelonka RL, Chai MK, Yoder BA, et al. Volume of blood required to detect common neonatal pathogens. J Pediatr 1996;129(2):275-278. DOI: 10.1016/S0022-3476(96)70254-8.

19. Struthers $S$, Underhill $H$, Albersheim $S$, et al. A comparison of two versus one blood culture in the diagnosis and treatment of coagulase-negative staphylococcus in the neonatal intensive care unit. J Perinatol 2002;22(7):547-549. DOI: 10.1038/sj.jp.7210792.

20. Garges HP, Moody MA, Cotten CM, et al. Neonatal meningitis: what is the correlation among cerebrospinal fluid cultures, blood cultures, and cerebrospinal fluid parameters? Pediatrics 2006;117(4):1094-1100. DOI: 10.1542/peds.2005-1132.

21. Johnson CE, Whitwell JK, Pethe K, et al. Term newborns who are at risk for sepsis: are lumbar punctures necessary? Pediatrics 1997;99(4):E10. DOI: 10.1542/peds.99.4.e10.

22. Committee on Infectious Diseases, Committee on Fetus and Newborn, Baker CJ, et al. Policy statement-recommendations for the prevention of perinatal group B streptococcal (GBS) disease. Pediatrics 2011;128(3):611-616. DOI: 10.1542/peds.2011-1466.

23. Greenwood C, Morrow AL, Lagomarcino AJ, et al. Early empiric antibiotic use in preterm infants is associated with lower bacterial diversity and higher relative abundance of Enterobacter. J Pediatr 2014;165(1):23-29. DOI: 10.1016/j.jpeds.2014.01.010.

24. Alexander VN, Northrup V, Bizzarro MJ. Antibiotic exposure in the newborn intensive care unit and the risk of necrotizing enterocolitis. J Pediatr 2011;159(3):392-397. DOI: 10.1016/j.jpeds.2011.02.035.

25. Ohlsson A, Lacy JB. Intravenous immunoglobulin for preventing infection in preterm and/or low birth weight infants. Cochrane Database Syst Rev 2013;7:CD000361. DOI: 10.1002/14651858. CD000361.pub3.

26. Ohlsson A, Lacy JB. Intravenous immunoglobulin for suspected or proven infection in neonates. Cochrane Database Syst Rev 2015;3:CD001239. DOI: 10.1002/14651858.CD001239.pub5.

27. Carr R, Modi N, Doré C. G-CSF and GM-CSF for treating or preventing neonatal infections. Cochrane Database Syst Rev 2003;3:CD003066. DOI: 10.1002/14651858.CD003066.

28. Pammi M, Haque KN. Pentoxifylline for treatment of sepsis and necrotizing enterocolitis in neonates. Cochrane Database Syst Rev 2015;3:CD004205. DOI: 10.1002/14651858.CD004205.pub3. 